\title{
Association of serum apolipoprotein B with cerebrospinal fluid Alzheimer's disease biomarkers in patients with subjective cognitive decline
}

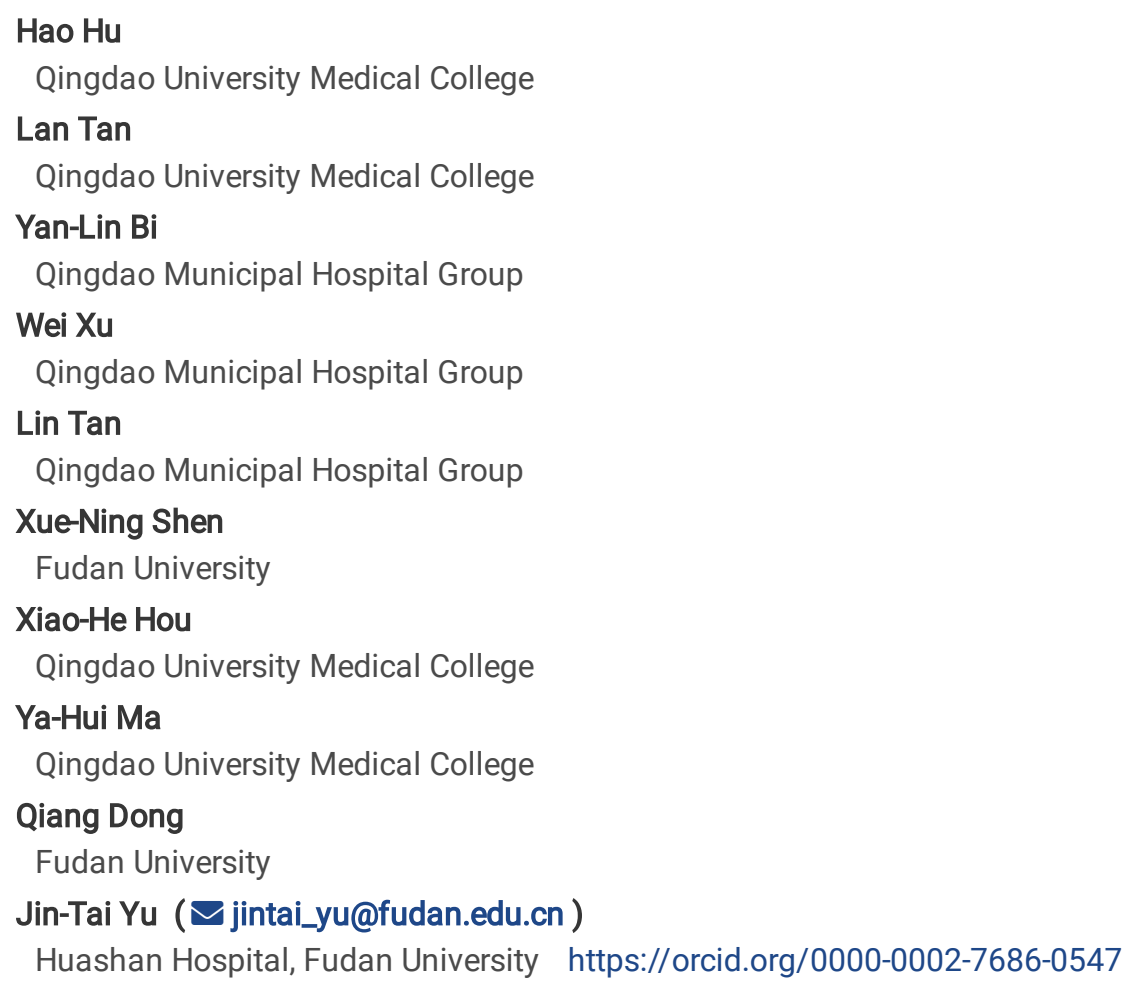




\section{Abstract}

Background The preclinical stage of Alzheimer's disease (AD) has become a key target stage for future AD prevention trials. Serum apolipoprotein, an important lipid-related factor, has been found to be involved in the pathogenesis of $A D$. This study was to examine whether apolipoprotein B (ApoB), apolipoprotein A-1 (ApoA1) or the ratio of ApoB and ApoA1 (ApoB/A1) were associated with early changes of cerebrospinal fluid (CSF) AD biomarkers in elderly adults with subjective cognitive decline.

Methods This study included 201 cognitive normal (CN) elderly adults and 101 participants with subjective cognitive decline (SCD) from the Chinese Alzheimer's Biomarker and LifestyIE (CABLE) database. The Kruskall-Wallis test and Chisquare test were applied in the intergroup comparisons. Multiple linear regression models were used to examine the cross-sectional associations of serum ApoB, ApoA1 and ApoB/A1 levels with CSF AD-related biomarkers.

Results Compared with the control group, SCD participants with significant AD biological characteristics had lower ApoB levels. In the total participants, higher level of serum ApoB was associated with increases in CSF AB42 ( $p=0.0009)$ and AB42/40 $(p=0.0038)$ as well as decreases in CSF t-tau/A $342(p<0.0001)$ and $p$-tau/A $342(p<0.0001)$, independent of APOE 4 status. In the further subgroup analysis, these associations were much more significant in the SCD participants. In addition, higher levels of serum ApoB were also found associated with decreases in CSF t-tau $(p=0.0224)$, $p$-tau $(p=0.0086)$ and A $\beta 40(p=0.0297)$ in the SCD subgroup. Furthermore, we found that these protective associations between serum ApoB and CSF AD core biomarkers were much more significant in the overweight participants. Results showed no association between ApoA1 and all CSF biomarkers in either total participants or subgroups.

Conclusions This study is the first to find protective associations of serum ApoB, but not ApoA1, with CSF AD core biomarkers in elderly adults, and these associations were more significant in SCD individuals. This finding indicated that ApoB may play a protective role in the preclinical stage of $A D$. Identifying the underlying mechanisms may contribute to the discovery of new pathogenic mechanisms and therapeutic targets.

\section{Background}

It has been widely accepted that neuropathological processes associated with Alzheimer's disease (AD) begin a decade or more before the emergence of obvious objective cognitive impairment [1-3]. Biomarker research proved that levels of cerebrospinal fluid (CSF) $\beta$ amyloid 42 (AB42), phosphorylated tau (p-tau) and total tau (t-tau) have been significantly abnormal in the preclinical stage of AD [3-5]. The 2018 National Institute on Aging and Alzheimer's Association (NIAA) research framework further included the three CSF biomarkers in the ATN framework, reflecting brain amyloidosis (A: AB42), tau pathology (T: p-tau) and neurodegeneration (N: t-tau) [6]. All these theories shifted the definition of $A D$ from a syndromic to a biological construct and leaded us to focus on disease's earlier stage such as preclinical stage.

Subjective cognitive decline (SCD) in individuals without objective cognitive impairment was recognized as a transitional stage between a fully asymptomatic stage and a mild cognitively impaired (MCl) stage of the disease [7, 8]. It has been suggested to be a possible first symptomatic expression of preclinical $A D$ and a target stage for future $A D$ prevention trials. Therefore, finding some available blood biomarkers associated with early pathological changes of $A D$ biomarkers in SCD individuals may contribute to creating more practical early diagnostic methods or finding some new factors involved in AD early pathogenesis.

Apolipoproteins are key proteins in the lipid metabolism of peripheral blood and central nervous system and also play an important role in the transport of some key proteins $[9,10]$. Previous studies have indicated that Apolipoprotein B (ApoB) and Apolipoprotein A-1 (ApoA1) may be involved in the pathogenesis of $A D$. Some studies have shown that $A D$ patients had higher levels of circulating ApoB and lower levels of circulating ApoA1 compared with healthy controls $[11,12]$. ApoB may promote the formation of amyloid plaques and vascular pathological changes $[13,14]$. ApoA1 may have a neuroprotective effect by forming a complex with the $A \beta$ peptide [12]. However, some studies showed different results [15]. Overall, the number of studies on these factors is still limited. Moreover, to our knowledge, no studies have been reported on the associations of serum ApoB, ApoA1 and the ratio of ApoB and ApoA1 (ApoB/A1) with CSF AD biomarkers, especially in the preclinical stage.

The purpose of our study was to examine whether $A p o B, A p o A 1$ and $A p o B / A 1$ can reflect the early pathological changes of CSF AD biomarkers in elderly adults with subjective cognitive decline and to find some potential candidate early biomarkers in blood for $A D$.

\section{Methods}




\section{Participants}

The participants without objective cognitive impairment in our study came from the Chinese Alzheimer's Biomarker and LifestylE (CABLE) database. The CABLE database is an independent replication cohort which has been approved by the Institutional Review Board of Qingdao Municipal Hospital. Since 2017, CABLE has been an ongoing large-scale study majorly focused on AD's risk factors and biomarkers in Chinese Northern Han population. CABLE aimed to determine the genetic and environmental modifiers of AD biomarkers and their utility in early diagnosis. The samples in CABLE study were recruited at Qingdao Municipal Hospital, consisting of cognitively normal individuals without subjective and objective cognitive decline (CN) and individuals with $S C D, M C l$ or AD.

The diagnosis of $A D$ dementia and $\mathrm{MCl}$ was made by experienced clinicians according to the criteria of National Institute on AgingAlzheimer's Association (NIA-AA) workgroups criteria [16, 17]. All clinical evaluations were conducted using standardized questionnaires. Specifically, the general cognitive function was assessed by a China-Modified Mini-Mental State Examination (CM-MMSE) and Montreal Cognitive Assessment (MoCA). The cut-off values after correcting the years of education were used in the MMSE $(\leq 24$ for $6+y$ of education, $\leq 20$ for 6 - $y$ of education, $\leq 17$ for $0 \mathrm{y}$ of education) and MOCA ( $<26$ for $12+y$ of education, $<25$ for $12-y$ of education). The basic living ability was assessed by basic Activities of Daily Living score (ADL). Behavioral or psychological symptoms were assessed by Geriatric Depression Scale (GDS), Hamilton Rating Scale for Depression (HAMD) and Hamilton Rating Scale for Anxiety (HAMA). The GDS and HAMD scores of participants in CABLE database were less than seven. Vascular factors were assessed by Hachinski Inchemic Score (HIS). The HIS scores of participants in our study were less than four. Subjective cognitive decline was assessed by a subjective cognitive decline scale (SCDS) which was designed based on SCD-I recommendations $[18,19]$. We distinguished participants with SCD accompanied by particular concerns (worries) from those without objective cognitive decline by the first section of SCDS which included a dichotomous question. Then we chose SCD participants who met the SCD-plus criteria as much as possible [19].

All enrolled participants in the CABLE were Han Chinese aged between 40 to 90 years old. The exclusion criteria include: (1) central nervous system infection, head trauma, epilepsy, multiple sclerosis or other major neurological disorders; (2) major psychological disorders; (3) severe systemic diseases; (4) family history of genetic diseases. All participants underwent biochemical testing as well as blood and CSF sample collection. Demographic information, AD risk factor profile and medical history were collected via a structured questionnaire and an electronic medical record system.

Finally, we included 302 participants without objective cognitive impairment from CABLE database, including 201 CN and 101 SCD participants. All the participants, who had no history of taking lipid-lowering drugs provided both blood and CSF samples.

\section{CSF sample collection and measurements}

Collection of fasting lumbar CSF samples and blood samples was performed at Qingdao Municipal Hospital. CSF was drawn and processed within two hours after collection. Each tube was centrifuged at 2000×g for 10 min, and CSF was separated and stored in enzyme free EP tube at -80 centigrade until further use in this study. The thaw/freezing cycle was limited and did not surpass two times. Baseline CSF Aß42, Aß40, t-tau, and p-tau181 were determined with the ELISA kit (Innotest $\beta$-AMYLOID (1-42), $\beta$-AMYLOID (1-40), hTAU$\mathrm{Ag}$, and PHOSPHO-TAU (181p); Fujirebio, Ghent, Belgium) on the microplate reader (Thermo Scientific Multiskan MK3). The within-batch coefficient of variation (CV) was $<5 \%$ (4.5\% for $A \beta 42,3.5 \%$ for $A \beta 40,4.4 \%$ for t-tau, and $2.5 \%$ for p-tau 181 ). The inter-batch CV was $<15 \%$ (9.6\% for $A \beta 42,8.1 \%$ for $A \beta 40,12.2 \%$ for $t$-tau, and $11.0 \%$ for $p$-tau 181 ).

\section{Blood sample collection and measurements}

Blood samples of all the participants were drawn after an overnight fast. Serum levels of ApoB and ApoA1 were assayed by immunonephelometry using a BN II analyzer (Siemens Healthcare, Marburg, Germany) within two hours after collection.

QIAamp ${ }^{\circledR}$ DNA Blood Mini Kit (250) was used to extract DNA from blood samples. And the extracted DNA was separated and stored in enzyme free EP tube at -80 centigrade until the apolipoprotein $\mathrm{E}(A P O E) 4$ genotyping was completed in this study. Specific loci were selected for genotyping with restriction fragment length polymorphism (RFLP) technology, including the two loci related to APOE4 status (rs7412 and rs429358).

\section{Standard protocol approvals, registrations, and patient consents}

The CABLE database was conducted in accordance with the Helsinki declaration, and the research program was approved by the Institutional Ethics Committee of Qingdao Municipal Hospital. All study participants or their caregivers provided written informed consent directly. 


\section{Statistical analysis}

Kruskall-Wallis test and Chisquare test were applied in the comparison among groups. Amyloid imaging and neuropathological studies showed that approximately one-third of older adults without objective cognitive impairment had AD pathology in their brains [20-23]. Therefore, in our study, CSF biomarker positive participants were defined as having CSF Aß42 levels in the lower one-third of the distribution of participants $(\mathrm{A}+: \leq 116.11 \mathrm{pg} / \mathrm{mL})$ or having p-tau $(\mathrm{T}+: \geq 39.17 \mathrm{pg} / \mathrm{mL})$ or t-tau $(\mathrm{N}+: \geq 180.59 \mathrm{pg} / \mathrm{mL})$ levels in the upper one-third of the distribution. All serum and CSF variables were log-transformed to normalize the distributions. Multiple linear regression models were used to examine the cross-sectional associations of baseline serum ApoB, ApoA1 and ApoB/A1 ratio with CSF biomarkers, adjusting for age, sex, education and APOE 4 status. The Bonferroni correction was used for multiple comparisons. Statistical analyses were conducted using $\mathrm{R}$, version 3.5.1. A two-tailed $\mathrm{p}<0.05$ was considered significant.

\section{Results}

\section{Characteristics of participants}

For the current study, we analyzed data from 302 participants of the CABLE cohort, including 201 CN and 101 SCD (see Table 1 for participants' characteristics). The average age of participants was 63 years; 111 (63\%) participants were male; and 40 (13\%) participants were $A P O E \triangle 4$ positive. We found no significant difference in age, sex distribution or level of education between two subgroups. Frequency of positive APOEE 4 status showed an increasing trend in SCD subgroup (CN: $11.94 \%$, SCD: $15.84 \%$ ), although these results were not statistically significant.

As for cognitive levels, we did not find a significant cognitive difference between CN subgroup and SCD subgroup (CM-MMSE: $p=0.3812$; MOCA: $p=0.0925)$. As for CSF biomarkers, compared with CN subgroup, SCD subgroup had lower levels of A $42(p=0.0080)$ and $A \beta 42 / 40(p<0.0001)$ and higher levels of $p$-tau $(p=0.0389)$, t-tau $/ A \beta 42(p=0.0004)$ and $p$-tau/Aß42 $(p=0.0011)$.

\section{Levels of serum ApoB and ApoA1 in different diagnostic groups}

As for levels of serum ApoB, in a clinical diagnostic construct, we did not find difference between $C N$ and SCD subgroups (Figure 1a). In an ATN biological construct, all CSF biomarker positive subgroups had lower levels of serum ApoB compared with CSF biomarker negative subgroups (Figure 1d, e, f). Then, according to the 2018 NIAA research framework, we chose $A+T-N-, A+T+N-$ and $A+T+N+$ as $A D$ continuum and chose $A-T-N$ - as control group. Results showed that $A+T+N+$ subgroup showed significantly lower levels of serum $A p o B$ than A-T-N- $(p=0.0009)$ (Figure 1b). Furthermore, in a diagnostic structure combining clinical diagnosis and biomarkers, SCD participants with $A+$ had significantly lower levels of serum ApoB than CN participants without pathologic changes in CSF biomarkers $(p=0.0093)$ (Figure 1c). As for ApoA1, we didn't find any difference in both clinical construct and biological construct (results were not shown).

\section{Associations of $A p \circ B, A p o A 1$, and ApoB/A1 with CSF AD core biomarkers in total participants without objective cognitive impairment}

The results on associations of $A p o B, A p o A 1$, and $A p o B / A 1$ with CSF biomarkers in total participants without objective cognitive impairment were shown in Table 2. Results showed that higher levels of serum ApoB were significantly associated with increased levels of CSF $A \beta 42(\beta=0.34, p=0.0009)$ and $A \beta 42 / 40(\beta=0.35, p=0.0038)$ but not with $A \beta 40$ (Table 2, Figure 2). Although no association of $A p o B$ was found with t-tau and $p$-tau, higher levels of serum ApoB were significantly associated with decreased levels of CSF t-tau/AB42 $(\beta=-0.47, p<0.0001)$ and $p$-tau/A $\beta 42(\beta=-0.41, p<0.0001)$ (Table 2, Figure 2). All results remained significant after Bonferroni

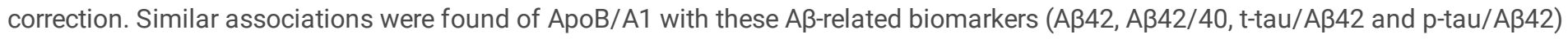
(Table 2), but these associations seemed less significant than those of ApoB (Table 2). We did not find any association between ApoA1 and all CSF biomarkers (Aß42, t-tau, p-tau, $A \beta 40, A \beta 42 / 40$, t-tau/Aß42 and p-tau/Aß42).

Furthermore, we added the interaction between $A P O E \otimes 4$ status and $A p o B$ into multiple linear regression models. Results showed that these associations between $\mathrm{ApoB}$ and $\mathrm{A} \beta$-related biomarkers were independent of $A P O E \varepsilon 4$ status. In these models, both $\mathrm{ApoB}$ and $A P O E \otimes 4$ status were associated with $A \beta$-related biomarkers, but their interactions ( $A p o B \times A P O E \varepsilon 4$ ) were not (see Additional file 1).

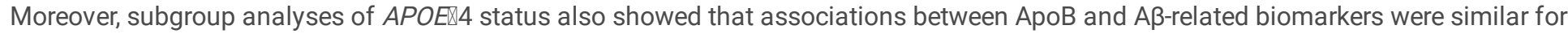
different $A P O E \otimes 4$ statuses (see Additional file 2).

\section{Associations of ApoB, ApoA1, and ApoB/A1 with CSF AD core biomarkers in different diagnostic subgroups}

Results from subgroup analyses of different diagnostic groups were shown in Table 3. As for ApoB, these associations between $A p o B$ and $A \beta$-related biomarkers (A $A 42, A \beta 42 / 40$, t-tau/A 42 and p-tau/A 42 ) still remained significant in both $C N$ and SCD subgroups (Table 
3 , Figure 3). It is worth noting that these associations were more significant in the SCD subgroup than in the CN subgroup (Table 3 ). In addition, we found that higher levels of ApoB were also associated with decreased CSF t-tau $(\beta=-0.37, p=0.0224), p$-tau $(\beta=-0.24, p=$ $0.0086)$ and $A \beta 40(\beta=-0.43, p=0.0297)$ in SCD subgroup, while these associations were not found in the CN subgroup (Table 3 , Figure

3). As for $A p o B / A 1$, significant associations between $A p o B / A 1$ and all CSF biomarkers (A 42 , t-tau, $p$-tau, $A \beta 40, A \beta 42 / 40, t-t a u / A \beta 42$ and p-tau/AB42) were found only in the SCD subgroup but not in the CN subgroup (Table 3). We did not find any association between ApoA1

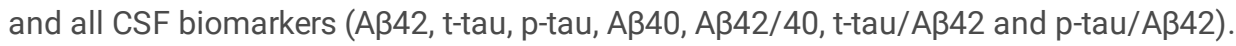

Given that there may be different metabolic states of apolipoproteins between overweight and normal populations, we further tested our results found above in different weight participants that were classified according to Body Mass Index (BMI) (normal weight participants: $\mathrm{BMI}<25$, overweight participants: BMI $\geq 25$ ). To our surprise, in both CN subgroup and SCD subgroup, almost all of associations found above existed only in the overweight participants but not in normal weight participants (Table4, Table 5).

\section{Discussion}

This study is the first to systematically explore cross-sectional associations of CSF AD core biomarkers with ApoB, ApoA1, and ApoB/A1 in elderly adults without objective cognitive impairment. The main finding of this study was that serum ApoB may have some protective effects on CSF AD core biomarkers in elderly adults without objective cognitive impairment independently of $A P O E \varepsilon 4$ genotype, especially in the SCD overweight population. This finding was novel and potentially important, because it suggested that levels of serum ApoB added information beyond APOE\&4 status on the level of CSF AD core biomarkers in the preclinical stage, which may contribute to the discovery of new pathogenic mechanisms and therapeutic targets.

First of all, we analyzed the characteristics of SCD participants included in our study. Results showed that, compared with the CN subgroup, although there was no significant cognitive decline, there were obvious changes in CSF AD core biomarkers (especially A $\beta$ related biomarkers) in the SCD subgroup, which were consistent with previous studies [8, 24]. In fact, accumulating cross-sectional or longitudinal evidence has supported that SCD occurred at the preclinical stage of $A D$ and might serve as a symptomatic indicator of preclinical $A D[7,8,24-30]$. Our analysis of population characteristics showed that, in our study, SCD participants had preclinical AD characteristics.

Then we explored the distribution of ApoB and ApoA in the diagnostic and biological constructs. Results showed that SCD participants with significant $A D$ biological characteristics had a lower ApoB level (Figure 1) suggesting that the level of ApoB may decrease in the preclinical stage of $A D$.

Finally we detailed the associations of $A p o B, A p o A 1$, and $A p o B / A 1$ with CSF AD core biomarkers in total participants or different diagnostic subgroups and analyzed the factors that might affect these associations. We firstly found that serum $A p o B$ and $A p o B / A 1$ were associated with CSF AD core biomarkers, especially with the $A \beta$-related biomarkers ( $A \beta 42, A \beta 42 / 40, t-t a u / A \beta 42$ and $p$-tau/A $A 42)$. It is worth noting that these associations were much more significant in SCD participants. Surprisingly, inconsistent with previous studies, above associations showed that serum $A p o B$ and $A p o B / A 1$ appeared to have some protective effects that may alleviate the early pathological changes of $A D$. To be precise, higher $A p o B$ and $A p o B / A 1$ were associated with increased CSF $A B 42$ and $A \beta 42 / 40$, as well as with decreased CSF t-tau, p-tau, $A \beta 40$, t-tau/A 42 and $p$-tau/A 442 . No previous studies have focused on the associations of serum $A p o B$ and $\mathrm{ApoB} / \mathrm{A} 1$ with CSF AD core biomarkers in cognitively normal or SCD participant. However, two autopsy studies in AD patients indicated that $A p o B$ may be a risk factor for the late stage of $A D$. One showed that serum ApoB were positive associated with the amount of $A \beta 42$ in AD brains [14]. The other showed that ApoB immunoreactivity was positively associated with cerebral amyloids [13]. The exact causes of these seemingly contradictory results were still unclear, but we thought that there were several possible reasons as follows.

Firstly, the different stages of the disease might contribute to this contradiction. Previous studies have found that changes in the bloodbrain barrier contributed differently to the dynamic changes of CSF AD core biomarkers in different stages of disease. CSF AD core biomarker (especaially A 42 ) may be temporarily elevated and its significant deposition in brain may occur when the blood-brain barrier was significantly impaired [31]. As a possible transporter of CSF biomarkers $₫ A p o B$ may facilitate the processes of early clearance and late deposition of biomarkers in brain. In addition, in the late stage of the disease, atherosclerosis and other vascular damages caused by ApoB-LDL may also aggravate the pathological changes, which may not be apparent in the early stage of the disease.

Secondly, genetic factors may also contribute to this contradiction. In this study, we tested the influences of $A P O E \varepsilon 4$ status on above associations. We found that these protective associations were independent of the APOEE 4 status, based on the following evidences. a) Both serum ApoB and APOE\&4 status were associated with CSF AD core biomarkers, but their interaction was not associated with these 
biomarkers. b) The results of the subgroups analysis were similar among population with different $A P O E \varepsilon 4$ status. This independence on $A P O E \varepsilon 4$ was also seen in a previous study on the association between serum $A p o B$ and amount of $A \beta 42$ in $A D$ brains [14]. Collectively, these data provided evidences that $A p \circ B$ and $A P O E \varepsilon 4$ status may contribute to the changes of CSF AD core biomarkers, independently of each other. However, a recent study found that rare genetic coding variants of $A P O B$ gene were strongly associated with familial $A D$, which indicated that $A P O B$ gene might also harbor protective and deleterious variants in sporadic AD similar to the $A P O E \varepsilon 2$ and $A P O E \varepsilon 4$ alleles [32]. Identifying such a protective coding variant of $A P O B$ gene would greatly strengthen the link between $A p o B$ and $A D$ pathogenesis.

In addition, some other factors may also contribute to this contradiction, such as changes involved low density lipoprotein cholesterol metabolism (the low density lipoprotein receptor and proprotein convertase subtilisin/kexin type 9). So given the concept that normal weight populations and overweight populations often have different lipid metabolism status, we further tested our results found above in different weight participants. It is worth noting that these protective effects of serum ApoB on CSF AD core biomarkers were particularly stronger in overweight participants compared with the normal weight participants. These results indicated that different lipid metabolism status may also influence above associations, which may contribute to the contradiction. Moreover, growing body of studies suggested that overweight in older age may be a protective factor for $A D$. Overweight older adults had a lower incidence or a later onset of $A D$ than those of normal weight or low weight [33-35]. Our results indicated that the protective effect of serum ApoB on CSF AD core biomarkers may be the potential mechanism. In other words, the serum ApoB's ability to alleviate early pathological changes may contribute to slow the progression of the disease in overweight older adults, but not in normal weight older adults.

Overall, we found protective associations of serum ApoB with CSF AD core biomarkers, these associations may be affected by disease's stages and lipid metabolism status or some other indefinite factors such as genetic factors. In this study we did not find any associations between ApoA1 and CSF AD core biomarkers.

However, a recent liquid chromatography-tandem mass spectrometry based proteomics study highlighted plasma ApoA1 as a biomarker in Alzheimer's disease and $A \beta$ burden, even in cognitively unimpaired individuals [36]. So the effects of ApoA1 on the early pathological changes in population without objective cognitively impairment still need to be tested in more studies.

There were some potential limitations in our study. Firstly, this was a cross-sectional study and the results still needed to be tested in larger longitudinal cohort. Secondly, the sample sizes of some subgroups were limited, which may affect the results to some extent.

\section{Conclusions}

This study was the first to find some protective associations of serum ApoB, but not ApoA1, with CSF AD core biomarkers in the preclinical stage of $A D$. This finding indicated that $A p o B$ may play different roles in different stages of $A D$. Further studies to identify the underlying mechanisms would greatly strengthen the link between $A p o B$ and $A D$ pathogenesis, which may contribute to the discovery of new pathogenic mechanisms and therapeutic targets.

\section{Abbreviations}

AD: Alzheimer disease; ADL: Activities of Daily Living score; AB42: $\beta$-amyloid 42; ApoA1: Apolipoprotein A-1; ApoB: Apolipoprotein B; APOE: apolipoprotein E; BMI: Body Mass Index; CABLE: Chinese Alzheimer's Biomarker and LifestyIE; CM-MMSE: China-Modified MiniMental State Examination; CSF: cerebrospinal fluid; GDS: Geriatric Depression Scale; HAMA: Hamilton Rating Scale for Anxiety; HAMD: Hamilton Rating Scale for Depression; HIS: Hachinski Inchemic Score; MCl: mild cognitively impaired; MoCA: Montreal Cognitive Assessment; NIAA: National Institute on Aging and Alzheimer's Association; p-tau: phosphorylated tau; SCD: Subjective cognitive decline; t-tau: total tau;

\section{Declarations}

\section{Acknowledgements}

Colleagues who have made contributions to build the CABLE cohort.

\section{Funding}


This study was supported by grants from the National Natural Science Foundation of China (91849126), the National Key R\&D Program of China (2018YFC1314700), Shanghai Municipal Science and Technology Major Project (No.2018SHZDZX01) and ZHANGJIANG LAB, Tianqiao and Chrissy Chen Institute, and the State Key Laboratory of Neurobiology and Frontiers Center for Brain Science of Ministry of Education, Fudan University.

\section{Authors' contributions}

$\mathrm{HH}$, Lan Tan, YLB, and JTY did the manuscript preparation and drafting. HH, LT, YLB, XW, XNS, XHH, YHM, and JTY did the clinical assessments and data acquisition. LT and JTY did the clinical diagnosis. HH, LT, YLB, XW, XNS, XHH, YHM, QD, and JTY did the data analysis and interpretation. JTY is responsible for the study conception and design. All authors have contributed to the manuscript revising and editing critically for important intellectual content and given final approval of the version and agreed to be accountable for all aspects of the work presented here.

\section{Availability of data and materials}

The datasets used and/or analysed during the current study are available from the corresponding author on reasonable request.

\section{Ethics approval and consent to participate}

The study was approved by the local ethics committee, and all individuals gave written informed consent to participate.

\section{Consent for publication}

Not applicable.

\section{Competing interests}

The authors declare that they have no competing interests

\section{Reference}

1. Selkoe, D.J. and J. Hardy, The amyloid hypothesis of Alzheimer's disease at 25 years. EMBO Mol Med, 2016. 8(6): p. 595-608.

2. Lane, C.A., J. Hardy, and J.M. Schott, Alzheimer's disease. Eur J Neurol, 2018. 25(1): p. 59-70.

3. Weiner, M.W., et al., The Alzheimer's disease neuroimaging initiative: progress report and future plans. Alzheimers Dement, 2010. 6(3): p. 202-11 e7.

4. Olsson, B., et al., CSF and blood biomarkers for the diagnosis of Alzheimer's disease: a systematic review and meta-analysis. Lancet Neurol, 2016. 15(7): p. 673-684.

5. Molinuevo, J.L., et al., Current state of Alzheimer's fluid biomarkers. Acta Neuropathol, 2018. 136(6): p. 821-853.

6. Jack, C.R., Jr., et al., NIA-AA Research Framework: Toward a biological definition of Alzheimer's disease. Alzheimers Dement, 2018. 14(4): p. 535-562.

7. Glodzik-Sobanska, L., et al., Subjective memory complaints: presence, severity and future outcome in normal older subjects. Dement Geriatr Cogn Disord, 2007. 24(3): p. 177-84.

8. Wolfsgruber, S., et al., Prevalence of abnormal Alzheimer's disease biomarkers in patients with subjective cognitive decline: crosssectional comparison of three European memory clinic samples. Alzheimers Res Ther, 2019. 11(1): p. 8.

9. Brown, M.S. and J.L. Goldstein, How LDL receptors influence cholesterol and atherosclerosis. Sci Am, 1984. 251(5): p. 58-66.

10. Emerging Risk Factors, C., et al., Lipid-related markers and cardiovascular disease prediction. JAMA, 2012. 307(23): p. $2499-506$.

11. Raygani, A.V., et al., Association between apolipoprotein E polymorphism and serum lipid and apolipoprotein levels with Alzheimer's disease. Neurosci Lett, 2006. 408(1): p. 68-72.

12. Merched, A., et al., Decreased high-density lipoprotein cholesterol and serum apolipoprotein Al concentrations are highly correlated with the severity of Alzheimer's disease. Neurobiol Aging, 2000. 21(1): p. 27-30.

13. Namba, Y., H. Tsuchiya, and K. Ikeda, Apolipoprotein B immunoreactivity in senile plaque and vascular amyloids and neurofibrillary tangles in the brains of patients with Alzheimer's disease. Neurosci Lett, 1992. 134(2): p. 264-6. 
14. Kuo, Y.M., et al., Elevated low-density lipoprotein in Alzheimer's disease correlates with brain abeta 1-42 levels. Biochem Biophys Res Commun, 1998. 252(3): p. 711-5.

15. Tynkkynen, J., et al., Apolipoproteins and HDL cholesterol do not associate with the risk of future dementia and Alzheimer's disease: the National Finnish population study (FINRISK). Age (Dordr), 2016. 38(5-6): p. 465-473.

16. Albert, M.S., et al., The diagnosis of mild cognitive impairment due to Alzheimer's disease: recommendations from the National Institute on Aging-Alzheimer's Association workgroups on diagnostic guidelines for Alzheimer's disease. Alzheimers Dement, 2011. 7(3): p. 270-9.

17. McKhann, G.M., et al., The diagnosis of dementia due to Alzheimer's disease: recommendations from the National Institute on AgingAlzheimer's Association workgroups on diagnostic guidelines for Alzheimer's disease. Alzheimers Dement, 2011. 7(3): p. 263-9.

18. Jessen, F., et al., A conceptual framework for research on subjective cognitive decline in preclinical Alzheimer's disease. Alzheimers Dement, 2014. 10(6): p. 844-52.

19. Molinuevo, J.L., et al., Implementation of subjective cognitive decline criteria in research studies. Alzheimers Dement, 2017. 13(3): p. 296-311.

20. Reiman, E.M., et al., Fibrillar amyloid-beta burden in cognitively normal people at 3 levels of genetic risk for Alzheimer's disease. Proc Natl Acad Sci U S A, 2009. 106(16): p. 6820-5.

21. Bennett, D.A., et al., Neuropathology of older persons without cognitive impairment from two community-based studies. Neurology, 2006. 66(12): p. 1837-44.

22. Hulette, C.M., et al., Neuropathological and neuropsychological changes in "normal" aging: evidence for preclinical Alzheimer disease in cognitively normal individuals. J Neuropathol Exp Neurol, 1998. 57(12): p. 1168-74.

23. Knopman, D.S., et al., Neuropathology of cognitively normal elderly. J Neuropathol Exp Neurol, 2003. 62(11): p. 1087-95.

24. Miebach, L., et al., Which features of subjective cognitive decline are related to amyloid pathology? Findings from the DELCODE study. Alzheimers Res Ther, 2019. 11(1): p. 66.

25. Jessen, F., et al., Prediction of dementia by subjective memory impairment: effects of severity and temporal association with cognitive impairment. Arch Gen Psychiatry, 2010. 67(4): p. 414-22.

26. Reisberg, B., et al., Outcome over seven years of healthy adults with and without subjective cognitive impairment. Alzheimers Dement, 2010. 6(1): p. 11-24.

27. Wang, Y., et al., Altered default mode network connectivity in older adults with cognitive complaints and amnestic mild cognitive impairment. J Alzheimers Dis, 2013. 35(4): p. 751-60.

28. Perrotin, A., et al., Subjective cognition and amyloid deposition imaging: a Pittsburgh Compound B positron emission tomography study in normal elderly individuals. Arch Neurol, 2012. 69(2): p. 223-9.

29. Prichep, L.S., et al., Prediction of longitudinal cognitive decline in normal elderly with subjective complaints using electrophysiological imaging. Neurobiol Aging, 2006. 27(3): p. 471-81.

30. van Harten, A.C., et al., Cerebrospinal fluid Abeta42 is the best predictor of clinical progression in patients with subjective complaints. Alzheimers Dement, 2013. 9(5): p. 481-7.

31. Potter, R., et al., Increased in vivo amyloid-beta42 production, exchange, and loss in presenilin mutation carriers. Sci Transl Med, 2013. 5(189): p. 189ra77.

32. Wingo, T.S., et al., Association of Early-Onset Alzheimer Disease With Elevated Low-Density Lipoprotein Cholesterol Levels and Rare Genetic Coding Variants of APOB. JAMA Neurol, 2019. 76(7): p. 809-817.

33. Hughes, T.F., et al., Association between late-life body mass index and dementia: The Kame Project. Neurology, 2009. 72(20): p. 17416.

34. Lee, J.Y., et al., Risk of Incident Dementia According to Metabolic Health and Obesity Status in Late Life: A Population-Based Cohort Study. J Clin Endocrinol Metab, 2019. 104(7): p. 2942-2952.

35. Tolppanen, A.M., et al., Midlife and late-life body mass index and late-life dementia: results from a prospective population-based cohort. J Alzheimers Dis, 2014. 38(1): p. 201-9.

36. Westwood, S., et al., Blood-Based Biomarker Candidates of Cerebral Amyloid Using PiB PET in Non-Demented Elderly. J Alzheimers Dis, 2016. 52(2): p. 561-72.

\section{Tables}

Page $8 / 15$ 
Table 1. Characteristics of study participants from CABLE database

\begin{tabular}{|c|c|c|c|c|}
\hline Variable & $\mathrm{CN}$ & SCD & Total & $\mathbf{P}$ \\
\hline $\mathbf{N}$ & 201 & 101 & 302 & - \\
\hline AGE (year) mean (SD) & $62.08(11.42)$ & $64.37(10.00)$ & $62.85(11.00)$ & $0.0783^{\#}$ \\
\hline SEX (Female/Male) & $71 / 130$ & $40 / 61$ & $111 / 191$ & $0.5475^{*}$ \\
\hline Education (year) mean (SD) & $9.90(4.49)$ & $10.49(4.34)$ & $10.09(4.44)$ & $0.2427^{\#}$ \\
\hline$A P O E \varepsilon 4 \mathrm{~N}(\%)$ & $24(11.94)$ & $16(15.84)$ & $40(13.25)$ & $0.4450^{*}$ \\
\hline CSF AB42 (pg/mL) mean (SD) & $181.96(130.45)$ & $149.75(81.13)$ & $171.19(117.16)$ & $0.0080^{\#}$ \\
\hline CSF t-tau $(\mathrm{pg} / \mathrm{mL})$ mean $(\mathrm{SD})$ & $175.83(82.36)$ & $186.86(85.62)$ & 179.52 (83.49) & $0.0509^{\#}$ \\
\hline CSF p-tau (pg/mL) mean (SD) & $37.55(9.90)$ & $39.02(8.55)$ & $38.04(9.48)$ & $0.0389^{\#}$ \\
\hline CSF AB40 (pg/mL) mean (SD) & $6525.83(3070.02)$ & $6998.23(2339.81)$ & $6683.82(2851.52)$ & $0.1386^{\#}$ \\
\hline CSF AB42/40 mean (SD) & $0.03(0.03)$ & $0.02(0.01)$ & $0.03(0.03)$ & $<0.0001^{\#}$ \\
\hline CSF t-tau/AB42 mean (SD) & $1.16(0.69)$ & $1.57(1.07)$ & $1.30(0.86)$ & $0.0004^{\#}$ \\
\hline CSF p-tau/Aß42 mean (SD) & $0.25(0.10)$ & $0.30(0.13)$ & $0.27(0.11)$ & $0.0011^{\#}$ \\
\hline CM-MMSE mean (SD) & $27.82(2.13)$ & $27.50(2.50)$ & $27.71(2.26)$ & $0.3812^{\#}$ \\
\hline MOCA mean (SD) & $26.92(0.99)$ & $26.74(0.98)$ & $26.86(0.99)$ & $0.0925^{\#}$ \\
\hline ApoB (mg/mL) mean (SD) & $1.04(0.25)$ & $1.02(0.27)$ & $1.03(0.26)$ & $0.5097^{\#}$ \\
\hline ApoA1 (mg/mL) mean (SD) & $1.45(0.30)$ & $1.43(0.28)$ & $1.45(0.29)$ & $0.9588^{\#}$ \\
\hline ApoB/A1 mean (SD) & $0.74(0.20)$ & $0.74(0.25)$ & $0.74(0.22)$ & $0.7560^{\#}$ \\
\hline BMI mean (SD) & $25.51(3.28)$ & $25.24(3.25)$ & $25.34(3.40)$ & $0.8450^{\#}$ \\
\hline
\end{tabular}

Abbreviations: $\mathrm{CN}$, cognitively normal participants; SCD, participants with subjective cognitive decline; $A P O E$, apolipoprotein E gene; CSF, cerebrospinal fluid; $A \beta$, amyloid- $\beta$; $t$-tau, total tau protein; -tau, phosphorylated tau protein; CM-MMSE, China-Modified Mini-Mental State Examination; MOCA, Montreal Cognitive Assessment; ApoB, Apolipoprotein B; ApoA1, Apolipoprotein A-1; BMI, Body Mass Index; SD, standard deviation

Bold indicated that the results were statistically significant.

\# Intergroup comparisons were tested by Kruskall-Wallis test.

* Intergroup comparisons were tested by Chisquare test.

Table 2. Results of cross-sectional associations in total participants 


\begin{tabular}{|c|c|c|c|c|c|c|}
\hline \multirow[t]{2}{*}{ Variable } & \multicolumn{2}{|c|}{ ApoB } & \multicolumn{2}{|c|}{ ApoA1 } & \multicolumn{2}{|c|}{ ApoB/A1 } \\
\hline & $\beta$ & $P$ & $\beta$ & $\mathbf{P}$ & $\beta$ & $\mathbf{P}$ \\
\hline CSF A $\mathrm{B} 42$ & 0.34 & 0.0009 & 0.07 & 0.5930 & 0.22 & 0.0135 \\
\hline CSF t-tau & -0.13 & 0.1556 & -0.06 & 0.6207 & -0.07 & 0.3656 \\
\hline CSF p-tau & -0.07 & 0.1823 & -0.01 & 0.9055 & -0.05 & 0.2823 \\
\hline CSF AB40 & -0.06 & 0.5620 & -0.03 & 0.8170 & -0.03 & 0.7260 \\
\hline CSF AB42/40 & 0.35 & 0.0038 & 0.17 & 0.2920 & 0.19 & 0.0179 \\
\hline CSF t-tau/Aß42 & -0.47 & $<0.0001$ & -0.13 & 0.3918 & -0.29 & 0.0034 \\
\hline CSF p-tau/AB42 & -0.41 & $<0.0001$ & -0.08 & 0.5331 & -0.27 & 0.0015 \\
\hline
\end{tabular}

Abbreviations: $C S F$, cerebrospinal fluid; $A \beta$, amyloid- $\beta$; t-tau, total tau protein; $p$-tau, phosphorylated tau protein; $A p o B$, Apolipoprotein B; ApoA1, Apolipoprotein A-1

Bold indicated that the results were statistically significant.

Multiple linear regression models were used to examine the crosssectional associations between baseline serum ApoB, ApoA1 and ApoB/A1 levels with CSF biomarkers, adjusting for age, sex, education and APOE\4 status.

Table 3. Results of cross-sectional associations in different diagnostic subgroups

\begin{tabular}{|c|c|c|c|c|c|c|c|c|c|c|c|c|}
\hline \multirow{3}{*}{ Variable } & \multicolumn{6}{|c|}{$\mathrm{CN}$} & \multicolumn{6}{|c|}{ SCD } \\
\hline & \multicolumn{2}{|c|}{ ApoB } & \multicolumn{2}{|c|}{ ApoA1 } & \multicolumn{2}{|c|}{ ApoB/A1 } & \multicolumn{2}{|c|}{ ApoB } & \multicolumn{2}{|c|}{ ApoA1 } & \multicolumn{2}{|c|}{ ApoB/A1 } \\
\hline & $\beta$ & $\mathbf{P}$ & $\beta$ & $\mathbf{P}$ & $\beta$ & $\mathbf{P}$ & $\beta$ & $\mathbf{P}$ & $B$ & $\mathbf{P}$ & $\beta$ & $\mathbf{P}$ \\
\hline CSF AB42 & 0.33 & 0.0133 & 0.06 & 0.7210 & 0.23 & 0.0531 & 0.50 & 0.0084 & 0.09 & 0.8010 & 0.39 & 0.0258 \\
\hline CSF t-tau & -0.07 & 0.5586 & -0.08 & 0.5941 & -0.01 & 0.8840 & -0.37 & 0.0224 & -0.19 & 0.5183 & -0.25 & 0.0110 \\
\hline CSF p-tau & -0.01 & 0.9937 & -0.04 & 0.6545 & 0.02 & 0.7604 & -0.24 & 0.0086 & -0.10 & 0.5762 & -0.17 & 0.0394 \\
\hline CSF AB40 & -0.02 & 0.8860 & -0.14 & 0.4460 & 0.05 & 0.6860 & -0.43 & 0.0297 & 0.01 & 0.9750 & -0.35 & 0.0489 \\
\hline CSF & 0.35 & 0.0150 & 0.20 & 0.2869 & 0.18 & 0.1622 & 0.70 & 0.0009 & 0.46 & 0.2510 & 0.46 & 0.0208 \\
\hline $\mathrm{AB} 42 / 40$ & & & & & & & & & & & & \\
\hline $\begin{array}{l}\text { CSF t- } \\
\text { tau/AB42 }\end{array}$ & -0.40 & 0.0051 & -0.14 & 0.4501 & -0.25 & 0.0524 & -0.87 & 0.0001 & -0.28 & 0.5200 & -0.64 & 0.0024 \\
\hline $\begin{array}{l}\text { CSF p- } \\
\text { tau/Aß42 }\end{array}$ & -0.33 & 0.0073 & -0.10 & 0.5296 & -0.21 & 0.0548 & -0.74 & $<0.0001$ & -0.19 & 0.6080 & -0.56 & 0.0011 \\
\hline
\end{tabular}

Abbreviations: CN, cognitively normal participants; SCD, participants with subjective cognitive decline; CSF, cerebrospinal fluid; $A \beta$, amyloid- $\beta$; t-tau, total tau protein; $p$-tau, phosphorylated tau protein; ApoB, Apolipoprotein B; ApoA1, Apolipoprotein A-1

Bold indicated that the results were statistically significant.

Multiple linear regression models were used to examine the crosssectional associations between baseline serum ApoB, ApoA1 and ApoB/A1 levels with CSF biomarkers, adjusting for age, sex, education and APOE\4 status. 
Table 4. Results of cross-sectional associations among different weight participants in CN subgroup

\begin{tabular}{|c|c|c|c|c|c|c|c|c|c|c|c|c|}
\hline \multirow{3}{*}{ Variable } & \multicolumn{6}{|c|}{ Normal-weight $(\mathrm{BMI}<25) \mathrm{N}=89$} & \multicolumn{6}{|c|}{ Overweight (BMI $\geq 25) \mathrm{N}=114$} \\
\hline & \multicolumn{2}{|c|}{ ApoB } & \multicolumn{2}{|c|}{ ApoA1 } & \multicolumn{2}{|c|}{ ApoB/A1 } & \multicolumn{2}{|c|}{ ApoB } & \multicolumn{2}{|c|}{ ApoA1 } & \multicolumn{2}{|c|}{ ApoB/A1 } \\
\hline & $\beta$ & $P$ & $\beta$ & $P$ & $\beta$ & $P$ & $\beta$ & $P$ & $\beta$ & $P$ & $\beta$ & $P$ \\
\hline CSF A $\mathrm{B} 42$ & 0.16 & 0.3920 & 0.28 & 0.2090 & -0.03 & 0.8430 & 0.39 & 0.0395 & -0.09 & 0.7390 & 0.36 & 0.0371 \\
\hline CSF t-tau & 0.03 & 0.8749 & 0.10 & 0.6211 & -0.03 & 0.8163 & -0.13 & 0.4190 & -0.28 & 0.1989 & 0.02 & 0.9070 \\
\hline CSF p-tau & -0.01 & 0.9330 & 0.07 & 0.5612 & -0.04 & 0.6141 & 0.02 & 0.8650 & -0.15 & 0.2670 & 0.08 & 0.3750 \\
\hline CSF A $\beta 40$ & -0.19 & 0.3650 & 0.02 & 0.4180 & -0.24 & 0.1670 & 0.09 & 0.6580 & -0.42 & 0.1180 & 0.26 & 0.1510 \\
\hline CSF & 0.35 & 0.0847 & 0.09 & 0.7140 & 0.21 & 0.2320 & 0.30 & 0.1490 & 0.34 & 0.2360 & 0.10 & 0.6050 \\
\hline$A \beta 42 / 40$ & & & & & & & & & & & & \\
\hline $\begin{array}{l}\text { CSF t- } \\
\text { tau/Aß42 }\end{array}$ & -0.14 & 0.5080 & -0.18 & 0.4480 & -0.01 & 0.9970 & -0.52 & 0.0086 & -0.19 & 0.4885 & -0.34 & 0.0578 \\
\hline $\begin{array}{l}\text { CSF p- } \\
\text { tau/AB42 }\end{array}$ & -0.17 & 0.3580 & -0.21 & 0.3290 & -0.01 & 0.9510 & -0.37 & 0.0263 & -0.06 & 0.7971 & -0.28 & 0.0673 \\
\hline
\end{tabular}

Abbreviations: $\mathrm{CN}$, cognitively normal participants; BMI, Body Mass Index; N, number of samples; CSF, cerebrospinal fluid; $A \beta$, amyloid- $\beta$; t-tau, total tau protein; p-tau, phosphorylated tau protein; ApoB, Apolipoprotein B; ApoA1, Apolipoprotein A-1

Bold indicated that the results were statistically significant.

Multiple linear regression models were used to examine the crosssectional associations between baseline serum ApoB, ApoA1 and ApoB/A1 levels with CSF biomarkers, adjusting for age, sex, education and APOE\4 status.

Table 5. Results of cross-sectional associations among different weight participants in SCD subgroup 


\begin{tabular}{|c|c|c|c|c|c|c|c|c|c|c|c|c|}
\hline \multirow{3}{*}{ Variable } & \multicolumn{6}{|c|}{ Normal-weight $(\mathrm{BMI}<25) \mathrm{N}=49$} & \multicolumn{6}{|c|}{ Overweight (BMI $\geq 25) \mathrm{N}=52$} \\
\hline & \multicolumn{2}{|c|}{ ApoB } & \multicolumn{2}{|c|}{ ApoA1 } & \multicolumn{2}{|c|}{ ApoB/A1 } & \multicolumn{2}{|c|}{ ApoB } & \multicolumn{2}{|c|}{ ApoA1 } & \multicolumn{2}{|c|}{ ApoB/A1 } \\
\hline & $\beta$ & $\mathbf{P}$ & $\beta$ & $\mathbf{P}$ & $\beta$ & $P$ & $\beta$ & $\mathbf{P}$ & $\beta$ & $\mathbf{P}$ & $\beta$ & $P$ \\
\hline CSF & 0.08 & 0.8350 & 0.38 & 0.4610 & -0.08 & 0.7850 & 0.64 & 0.0052 & -0.59 & 0.2790 & 0.65 & 0.0017 \\
\hline$A \beta 42$ & & & & & & & & & & & & \\
\hline $\begin{array}{l}\text { CSF t- } \\
\text { tau }\end{array}$ & -0.41 & 0.2590 & -0.26 & 0.5900 & -0.15 & 0.5910 & -0.51 & 0.0012 & -0.46 & 0.2301 & -0.36 & 0.0189 \\
\hline $\begin{array}{l}\text { CSF p- } \\
\text { tau }\end{array}$ & -0.18 & 0.3280 & -0.05 & 0.8190 & -0.08 & 0.5420 & -0.32 & 0.0051 & -0.30 & 0.2740 & -0.23 & 0.0417 \\
\hline $\begin{array}{l}\text { CSF } \\
\mathrm{A} 340\end{array}$ & -0.62 & 0.1270 & -0.23 & 0.6755 & -0.29 & 0.3674 & -0.54 & 0.0099 & -0.29 & 0.5637 & -0.42 & 0.0366 \\
\hline $\begin{array}{l}\text { CSF } \\
A \beta 42 / 40\end{array}$ & 0.70 & 0.1410 & 0.61 & 0.3330 & 0.20 & 0.5910 & 0.77 & 0.0017 & 0.51 & 0.3998 & 0.58 & 0.0147 \\
\hline $\begin{array}{l}\text { CSF t- } \\
\text { tau/Aß42 }\end{array}$ & -0.50 & 0.3440 & -0.64 & 0.3470 & -0.07 & 0.8670 & -1.14 & $<0.0001$ & 0.13 & 0.8440 & -1.01 & $<0.0001$ \\
\hline $\begin{array}{l}\text { CSF p- } \\
\text { tau/AB42 }\end{array}$ & -0.26 & 0.4976 & -0.44 & 0.3760 & 0.01 & 0.9980 & -0.96 & $<0.0001$ & 0.29 & 0.6380 & -0.88 & $<0.0001$ \\
\hline
\end{tabular}

Abbreviations: SCD, participants with subjective cognitive decline; BMI, Body Mass Index; N, number of samples; CSF, cerebrospinal fluid; $A \beta$, amyloid- $\beta$; t-tau, total tau protein; $p$-tau, phosphorylated tau protein; ApoB, Apolipoprotein $B$; ApoA1, Apolipoprotein A-1

Bold indicated that the results were statistically significant.

Multiple linear regression models were used to examine the crosssectional associations between baseline serum ApoB, ApoA1 and ApoB/A1 levels with CSF biomarkers, adjusting for age, sex, education and APOE₫4 status.

\section{Figures}




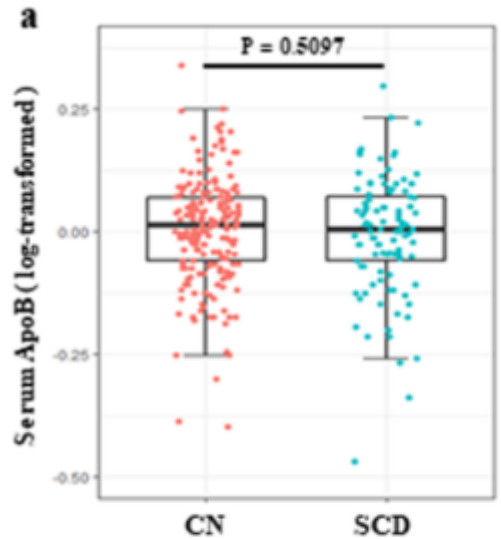

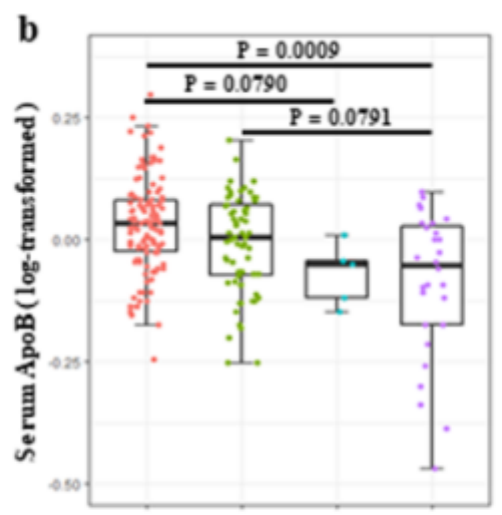

$\mathrm{A}-\mathrm{T}-\mathrm{N}-\quad \mathrm{A}+\mathrm{T}-\mathrm{N}-\quad \mathrm{A}+\mathrm{T}+\mathrm{N}-\mathrm{A}+\mathrm{T}+\mathrm{N}+$

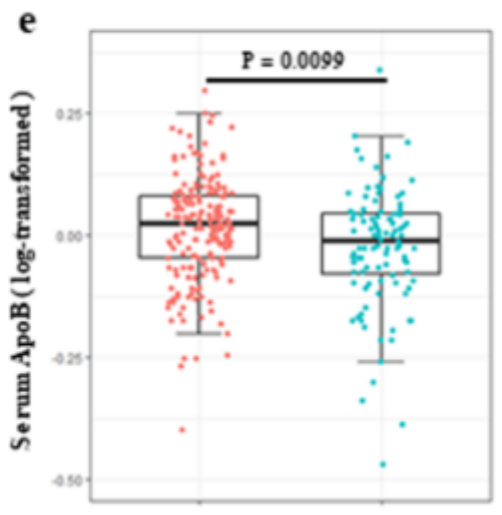

T-
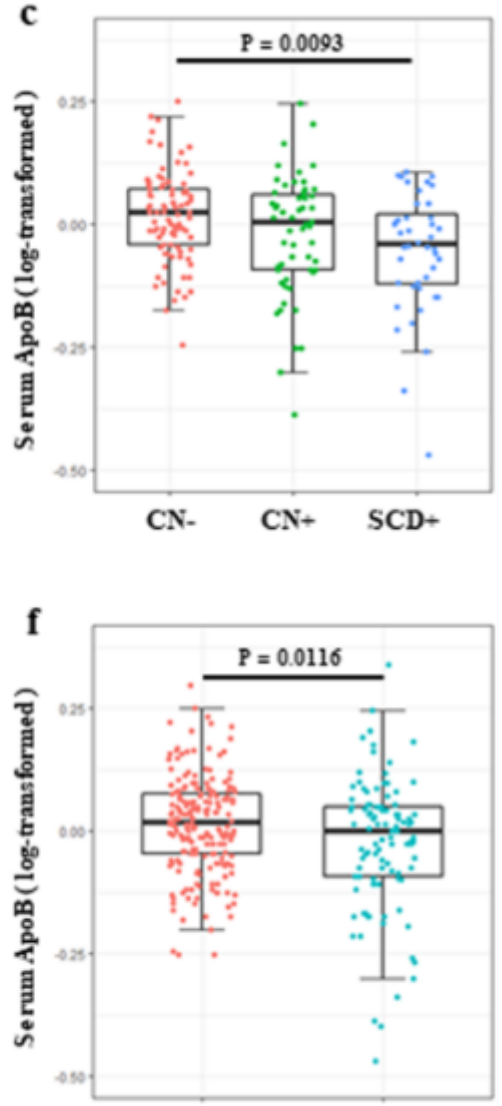

N-

$\mathrm{N}+$

\section{Figure 1}

Levels of serum ApoB in different groups (a) Levels of serum ApoB in a clinical diagnostic construct. $C N(N=201)$ : cognitively normal individuals without subjective and objective cognitive decline; SCD ( $N=101)$ : individuals with subjective cognitive decline; (b) Levels of serum ApoB in an ATN biological construct. A-T-N- $(N=120), A+T-N-(N=63), A+T+N-(N=5), A+T+N+(N=28)$; (c) Levels of serum ApoB in a diagnostic structure combining clinical diagnosis and biomarkers. $C N-(N=90)$ : cognitively normal individuals without subjective and objective cognitive decline and with three negative CSF biomarkers [A-T-N-]; $C N+(N=57)$ : cognitively normal individuals without subjective and objective cognitive decline and with positive CSF AB42 [A+]; SCD+ $(N=44)$ : individuals with subjective cognitive decline and positive CSF AB42 [A+]. (d) (e) (f) Levels of serum ApoB in A or T or N construct respectively. All intergroup comparisons were tested by Kruskall-Wallis test. 

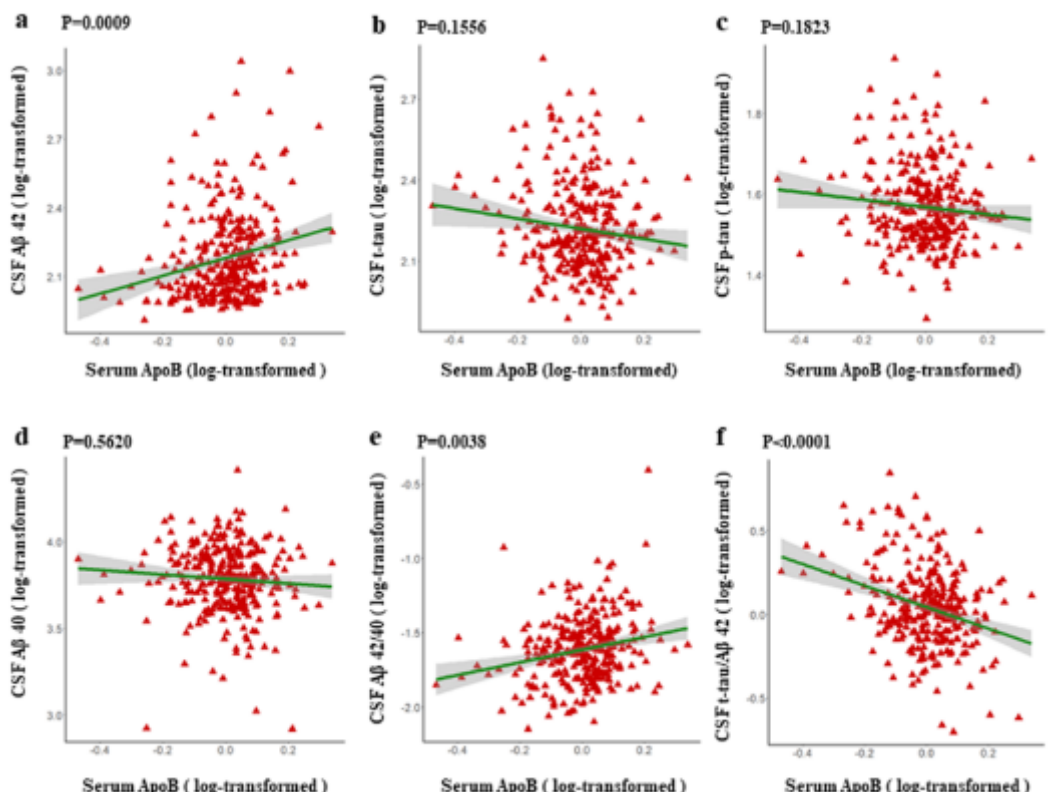

Serum ApoB ( log-transformed )

Serum ApoB ( log-transformed )

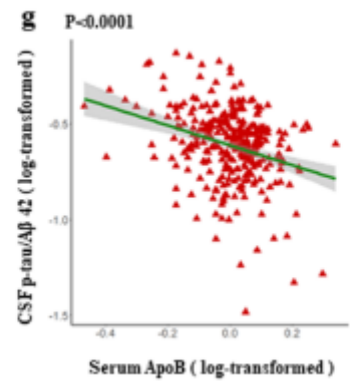

Figure 2

Associations between ApoB and CSF AD biomarkers in total participants Abbreviations: CSF, cerebrospinal fluid; $A \beta$, amyloid- $\beta$; $t$-tau, total tau protein; $p$-tau, phosphorylated tau protein; ApoB, Apolipoprotein B Multiple linear regression models were used to examine the crosssectional associations between baseline serum ApoB levels with CSF biomarkers, adjusting for age, sex, education and APOE 4 status. 

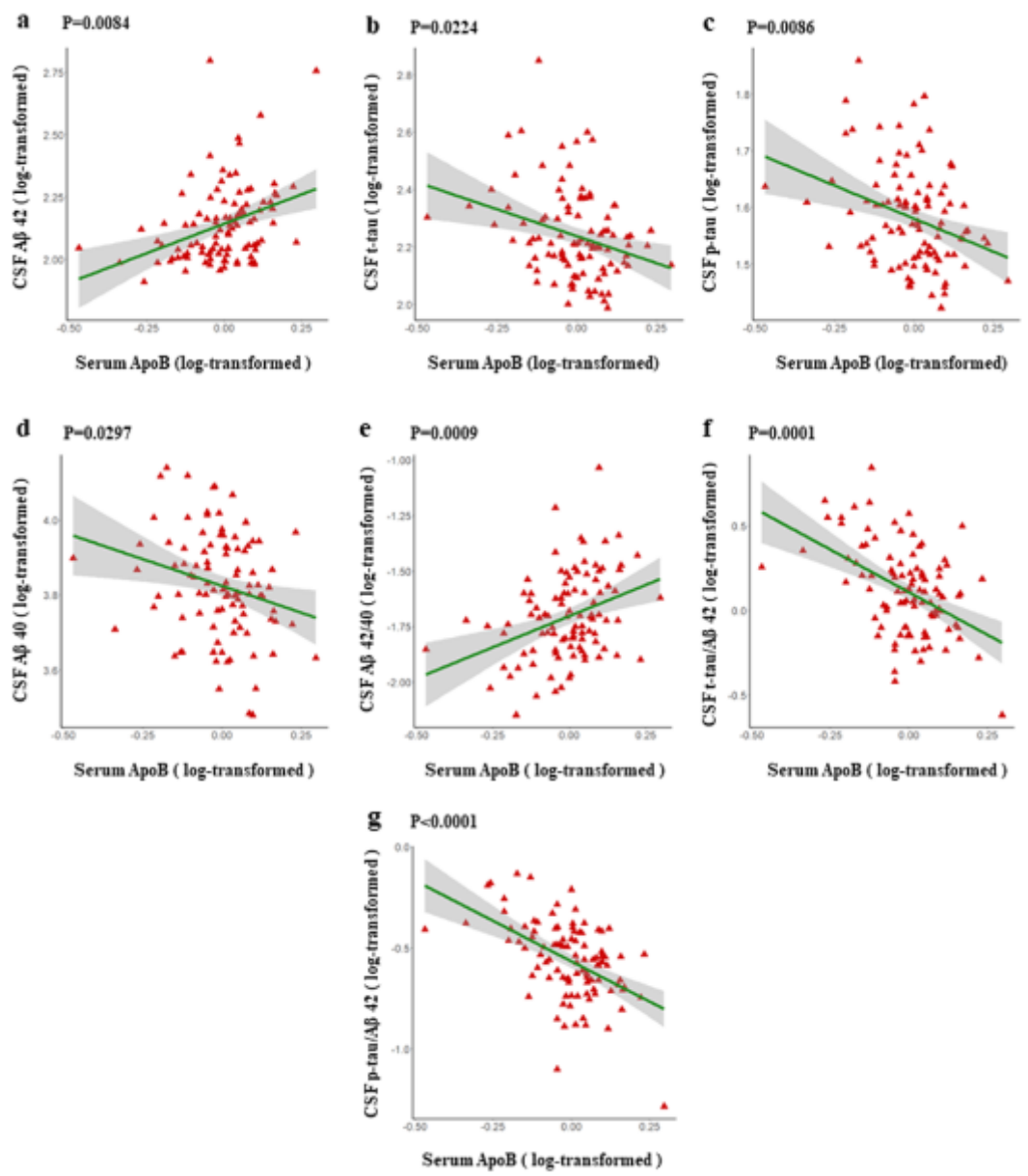

Figure 3

Associations between ApoB and CSF AD biomarkers in SCD participants Abbreviations: CSF, cerebrospinal fluid; $A \beta$, amyloid- $\beta$; t-tau, total tau protein; p-tau, phosphorylated tau protein; ApoB, Apolipoprotein B Multiple linear regression models were used to examine the crosssectional associations between baseline serum ApoB levels with CSF biomarkers, adjusting for age, sex, education and APOE 4 status.

\section{Supplementary Files}

This is a list of supplementary files associated with this preprint. Click to download.

- Additionalfile.docx 